\title{
Use of Eye Movement Tracking in the Differential Diagnosis of Attention Deficit Hyperactivity Disorder (ADHD) and Reading Disability
}

\author{
Pamela Deans ${ }^{1}$, Liz O’Laughlin², Brad Brubaker², Nathan Gay ${ }^{3}$, Damon Krug ${ }^{4}$ \\ ${ }^{1}$ Mule Creek State Prison, Ione, USA ${ }^{2}$ Psychology Department, Indiana State University, Terre Haute, USA, ${ }^{3}$ Devereux Foundation, \\ Rutland, USA, ${ }^{4}$ CDCSEP, Indiana State University, Terre Haute, USA. \\ Email: lizo@indstate.edu
}

Received July 16 ${ }^{\text {th }}$, 2010; revised August $2^{\text {nd }}$, 2010; accepted August $21^{\text {st }}, 2010$.

\begin{abstract}
The present study examined the clinical utility of eye movement tracking in the differential diagnosis of Attention Deficit/Hyperactivity Disorder (ADHD) and Reading Disorder (RD). It was anticipated that eye movement tracking would provide a better understanding of the underlying deficits that potentially contribute to reading difficulties among children with $A D H D$ and $R D$. Participants included 27 children diagnosed with ADHD, 20 that met criteria for a reading disorder and 30 Control children with no clinical diagnosis. All participants were between the ages of 6 to 12. Consistent with previous research, children in the $R D$ group displayed slower reading time, longer fixation duration and more atypical eye movements as compared to Control children. Children with ADHD also displayed more atypical eye movement as compared to Control children. The only significant difference between the ADHD and RD groups was in total reading time. Results of a discriminant analysis revealed that less than $60 \%$ of participants were given the correct diagnostic classification based on total reading time and proportion of left to right saccades indicating limited support for this measure in diagnosis of ADHD versus $R D$.
\end{abstract}

Keywords: Differential Diagnosis, Comorbidity, Attention Deficit/Hyperactivity Disorder, Reading Disability, Eye Movement Tracking

\section{Introduction}

Attention Deficit/Hyperactivity Disorder (ADHD) is one of the most commonly diagnosed disorders in children with prevalence rates in the general population ranging from 3-7\% [1]. Assessing a child for ADHD can be difficult given the subjectivity of currently utilized assessment measures and the high degree of comorbidity between ADHD and other disorders. Reading Disability (RD) is also commonly diagnosed with prevalence rates of approximately $4 \%$ among school-aged children in the general population [1]. Furthermore, there is a strong link between child behavior difficulties and underachievement in reading. For example, Nelson, Benner, Lane, and Smith (2004) found that 83\% of students classified with emotional and behavioral disorders performed in the below average range on a standardized measure of reading skills [2].

Although it is not unusual for children to meet criteria for both ADHD and RD (i.e., comorbidity estimates range from 10 to $45 \%$ ), children with RD may be misdiagnosed with ADHD and vice versa as it can be difficult to determine if a child displays reading problems due to behavioral difficulties or whether behavioral and attention difficulties are due to an underlying reading disorder. Misdiagnosis could lead to the implementation of inappropriate treatment interventions such as providing stimulant medication to a child with $\mathrm{RD}$ or providing academic interventions only to a child with ADHD. To reduce the likelihood of misdiagnosis and more clearly differentiate between ADHD and RD, an objective assessment measure is needed. One such instrument may be eye movement tracking. Eye movement tracking is a potentially useful method of assessment because it allows for objectivity and provides quantitative data on reading process and visual and attentional abilities [3].

\subsection{Assessment of ADHD and RD}

ADHD assessment typically involves the use of multiple 
methods and multiple informants. Information may be gathered through the use of diagnostic interviews, parent and teacher behavior rating scales, and direct observations of behavior. Other measures used in the assessment of ADHD include computerized performance tests and lab or analogue observations [4]. However, problems exist with each of these assessment tools. For example, diagnostic interviews and behavior rating scales are subjective, prone to social desirability, and are dependent upon accurate reporting of symptoms. Additionally, rating scales often contain vague and poorly defined items. Assessment methods such as computerized performance tests have been criticized due to poor clinical utility. Lab or analogue observations have also been criticized due to high subjectivity, as they are dependent upon the observer's criteria for what qualifies as inattentive or hyperactive behavior, and few observational measures have norms [5].

$\mathrm{RD}$ is a biologically based learning disorder that frequently runs in families and interferes with reading, writing, and spelling performance [6]. RD is characterized by difficulties in several areas of reading including decoding and spelling difficulties, comprehension problems, and deficits in phonological processing [7]. RD often affects a child's academic achievement and performance on other activities that require reading skills. Children with $\mathrm{RD}$ also exhibit distortions, substitutions, and omissions when reading aloud and silently [1]. Although symptoms of RD may be evident as early as kindergarten, it is rarely diagnosed before the first grade due to variations in when children begin acquiring reading skills.

The DSM-IV-TR criteria for a diagnosis of RD requires that an individual's reading achievement scores must be significantly lower than their overall level of intelligence as measured by an individually administered intelligence test [8]. However, the criteria used to classify a child for special education services based on a reading disability vary from state to state. Ideally, when assessing for RD an individual should be tested on the following skills: sight identification of letters and words, nonsense word decoding, spelling, reading comprehension, and reading fluency [6]. However, at the present time there are no individual tests or battery of tests designed specifically to evaluate for RD. Instead, examiners are required to develop their own assessment battery that includes measures of the necessary reading related skills [6]. Although examiners can diagnose RD by integrating the results of several measures, there is a need to develop a more comprehensive, valid, and objective method of diagnosing RD [9].

\subsection{Commonalities in Learning Difficulties}

In the school setting, students with ADHD often fall behind academically because of their attention problems. As a result of their poor academics, children with ADHD may appear to have a learning disability. Additional deficits that children with ADHD may display in the school setting include poor rote memory, excessive vocalizations, difficulty delaying gratification, distractibility by extraneous stimuli, and difficulty listening and maintaining a conversation [4]. These deficits, both individually and in combination, can make learning in the school setting very difficult. Thus, it is not surprising that children with ADHD are also more likely to be held back due to poor academic performance or suspended and expelled due to significant behavioral problems [10].

Children with RD often exhibit spoken language difficulties, deficits in short-term memory, poor reading comprehension, poor written expression, and difficulty organizing information [11]. Generally, children with RD perform poorly on tests of rapid automatized naming, phoneme awareness, sound blending, phonological skills, auditory memory, certain types of visual memory, and decoding of nonsense words [6]. A child with $\mathrm{RD}$ may also display symptoms of inattention in the classroom as he/she may be less academically engaged and may exhibit lower motivation to complete school related tasks. Thus, the potential for children with RD to be misdiagnosed with ADHD is significant if the symptoms of inattention are thought to be primary and the reading difficulties are considered secondary.

\subsection{Relationship between RD and ADHD}

With such high rates of comorbidity between ADHD and RD, some researchers do not consider ADHD to be a distinct clinical syndrome [12]. However, the bulk of the research clearly demonstrates that RD and ADHD represent two distinct clinical syndromes with separate cognitive profiles [8]. Children with RD exhibit deficiencies in phonological processing and other reading related skills while children with ADHD exhibit deficits in executive functioning. These unique and distinctive deficits provide support for the validity of each diagnosis.

\section{Eye Movement Tracking}

Eye movement tracking has also been used for a variety of purposes including diagnosing disorders related to reading and information processing abilities. When used for diagnostic purposes, eye movement tracking allows for objectivity and provides quantitative data on visual and attentional abilities [13]. Thus, eye movement tracking has great potential as a useful and scientifically valid method for studying patterns of reading for children with 
$\mathrm{RD}$ and attentional patterns for children with ADHD.

\subsection{Types of Eye Movements}

There are many different types of eye movements described in the literature. Following is a brief description of the eye movements that are most commonly observed during reading tasks. Fixations are movements made when the eye is relatively still and focused on a particular target. Fixations typically last between 200-300 milliseconds on average but can range from 100 to over 500 milliseconds. On tasks where the participant is asked to read silently, the average duration of a fixation is 225 milliseconds [14]. Saccades are defined as rapid movements that allow the eyes to move from one fixation point to another while scanning and processing the information between fixation points [14]. Saccades can range in length but are typically 7-9 letter spaces in silent reading [3]. Vertical saccades occur when the eye moves away from the target stimuli in an upward or downward fashion. This can occur when an individual becomes distracted and loses their attentional focus.

Regressions are small leftward saccades that are made when an individual has to re-read a section of text [14]. Regressions often occur when a saccade is too fast or cover more text than the individual can perceive. Approximately $10-15 \%$ of all saccades are regressions.

\subsection{Eye Movement and Reading}

Saccades and fixations are the two main types of eye movements involved in reading. However, the types of eye movements made by readers are significantly influenced by the qualities of the individual words and overall text. For example, fixations of longer duration tend to occur when a reader encounters words that are uncommon such as technical words, misspelled words, the first fixation of a new line, and parts of the text where the reader is anticipating important information. Likewise, fixations of shorter duration tend to occur on the final fixation of a line, at the beginning or end of a word, and immediately before a regression is made. Longer saccades tend to occur when a long word lies to the right of a fixation. Shorter saccades occur in parts of the text with important information because the reader needs to ensure that he/she has perceived all of the text [15].

Fixations are less likely to occur on shorter or more common words such as "the" and on blank areas of the text, and are more likely to occur near the center of a word. Additionally, words that contain content (e.g. dog, walk, hat, etc.) are fixated on more often than non-content words (e.g. the, is, and, etc.). As the length of a word increases, the probability of a fixation also increases because such words are harder to perceive during a saccade.
The first fixation on a line is typically 5-7 letter spaces from the beginning of a sentence while the last fixation occurs approximately 5-7 letter spaces from the end of a sentence. The first fixation also tends to be longer in duration while the last fixation of a line tends to be the shortest [3].

As the text becomes more difficult the number of fixations increase, saccade length decreases, and the number of regressions increase. Most readers make their first fixation on the first word of the sentence near the center of the word, but with long words (e.g. words with more than 10 letters) there are sometimes two fixations, one near the beginning of the word and the second towards the end of the word. Saccades that are too long tend to be less accurate and result in more regressions [3].

Kleigl \& Engbert [16] found that readers tend to skip over short, high frequency, and highly predictable words more often than long words, low frequency words, and words with low predictability. Kliegl \& Engbert [16] also noted fixations of shorter duration before words that were skipped. It is also important to note that the eye movements of a child are somewhat different from adult eye movements. For example, young children exhibit more frequent and small saccades, allow their eyes to drift during a fixation, take longer to initiate saccades, and are less accurate than adults in controlling their eye movements. As children age, their eye movements become more accurate and controlled [3].

\subsection{Eye Movements in Children with RD}

Previous research has demonstrated that children with $\mathrm{RD}$ exhibit different patterns of eye movement on reading tasks as compared to normal readers. While normal readers can read about 250 words per minute, the reading speed of children with RD tends to be much slower because they make longer fixations, more frequent fixations, shorter saccades, and more regressions than normal readers. Longer fixations often occur because it takes more time for these readers to comprehend information from the text. Children with RD also have shorter saccades because they cannot cover as much information in their perceptual span [17]. Additionally, children with $\mathrm{RD}$ tend to have unstable fixations and make more express (i.e., shorter) saccades than normals readers. Children with $\mathrm{RD}$ also process less parafoveal information (i.e. information in the periphery of the point of fixation) on each fixation leading to more frequent and shorter saccades [3]. Overall, these eye movement patterns are correlated with slower reading speed and poorer comprehension [15].

Eden, Stein, Wood \& Wood [14] found that eye movement tracking on non-reading tasks could reliably differ- 
entiate between good and poor readers. Poor readers tended to have jerky and erratic eye movements when attempting to visually track a moving target. Eden et al. [14] hypothesized that deficits in eye movement tracking among poor readers appear to be related to poor eye movement control.

Hawelka \& Wimmer [18] theorized that shorter saccades are the source of greater fixations among children with RD as compared to normal readers. Shorter saccades are common in letter-by-letter reading and contribute to a slow and laborious reading style. Hawelka \& Wimmer examined types of eye movements (e.g. fixations and saccades), speed of reading, and errors in reading in Dyslexic and Control children. Results of the study indicated that Dyslexic children made fewer errors than the Control group; however, their reading speed was significantly slower than the Control subjects. Differences in reading rate were associated with the number of eye movements made during reading. That is, participants with more eye movements had slower reading speeds [18]. These findings support the letter-by-letter reading pattern thought to be characteristic of reading disabled children.

\subsection{Eye Movements in Children with ADHD}

Munoz, Armstrong, Hampton, \& Moore [19] proposed that children and adults with ADHD may also have unique patterns of eye movement, particularly in regard to visual tracking tasks that require response inhibition of automatic saccadic eye movements. They utilized a prosaccade task in which ADHD and control participants ranging in age from 6 to 59 years old were asked to look at a target stimulus when it appeared on the screen and an antisaccade task where participants were asked to inhibit looking at the target stimulus. Results indicated that participants with ADHD displayed longer reaction times, more variability, and slower saccades in the prosaccade task compared to participants in the Control group. In the antisaccade task, participants with ADHD had more difficulty inhibiting automatic saccades, displayed longer reaction times, and greater variability [19].

Other researchers have found similar results suggesting that adults with ADHD exhibit different patterns of eye movement as compared to adults without ADHD. Feifel, Farber, Clementz, Perry, and Anllo-Vento [20] found that adult ADHD subjects made significantly more anticipatory saccades than Control subjects. On the antisaccade task, ADHD subjects made significantly more errors than the Control group. The performance of adults with ADHD was consistent with deficits in response inhibition seen in children with ADHD.

Gould, Bastain, Israel, Hommer, \& Castellanos [21] compared children with ADHD Combined type and Control children to determine if eye movement data could be used to provide objective criteria for diagnosing ADHD. The eye movement task required children to remain focused on a fixation point that was stable for a period of 30 seconds and then moved back and forth on a computer screen. Results indicated that children with ADHD had greater difficulty maintaining fixations and made more large saccades than the Control group. There were no gender or age differences. Gould et al. found that test-retest reliability of the eye movement task was poor as evidenced by the considerable variability in performance among the children across two testing periods two weeks apart. Gould et al. proposed that fixation errors have the potential to distinguish between children with and without ADHD; however, given the poor test-retest reliability this hypothesis is in need of further research. It should also be noted that this task required visual tracking ability only and not reading skills specifically.

Klein, Raschke \& Brandenbusch [22] also utilized an eye movement task that involved looking at a fixation point on a computer screen. They found that children with ADHD exhibited more errors and were also less likely to correct directional errors as compared to children in the Control group. However, group differences were dependent on age with older children displaying shorter response latencies and making fewer early (anticipatory) responses as compared to younger children.

Mostofsky, Lasker, \& Cutting [23] considered the influence of ADHD medication on eye movement performance. Both medicated and unmedicated children with ADHD made significantly more errors in direction and more anticipatory errors than did the Control children on the saccade tasks. These results are consistent with deficits related to ADHD such as poor response inhibition. Additionally, the unmedicated children showed greater variability in saccade latency on one task and longer saccade latency on a second task than the medicated and Control group. These results suggest that children with ADHD should ideally not be medicated when participating in studies utilizing eye movement in order to obtain data reflective of the true deficits associated with ADHD. However, group differences can still be detected even when children with ADHD are medicated.

The previous studies examining eye movement among children with ADHD all used eye movement paradigms that require tracking a visual stimulus rather than tasks that require reading skills. The present study sought to examine the eye movement patterns of children diagnosed with ADHD and RD during a reading task. It was anticipated that RD children would exhibit longer fixa- 
tion durations, more frequent fixations, and shorter saccades than children in the ADHD and Control groups.

It was also predicted that children with ADHD would exhibit a greater proportion of vertical saccades than children with $\mathrm{RD}$ or children in the Control group and that children in the Control group would exhibit a smaller proportion of regressive saccades, fewer fixations, and fixations of a briefer duration than children in the ADHD and RD groups.

\section{Method}

\subsection{Participants}

Children with ADHD and RD were recruited through a university-based ADHD Evaluation clinic and a separate reading intervention clinic (READ) both located in a moderate sized community in the Midwest. Children with ADHD were diagnosed by a licensed psychologist based on DSM-IV criteria [1] and using an assessment battery that included a structured interview, parent and teacher ratings on standardized measures, a computer-based test of attention and brief cognitive ability and academic achievement measures. Diagnosis of RD was made by a licensed School Psychologist who reviewed a battery of reading assessment measures administered by the READ clinic that included measures of phoneme awareness, phonics, reading fluency, and comprehension. Students were diagnosed with RD if scores were found to be in the impaired range in multiple assessed areas. Control participants were recruited through faculty and staff at the university. Data was collected from 110 participants between the ages of 6-12 years over a period of 30 months. However, as a result of equipment problems, invalid eye movement data due to excessive head or body movement and failure to meet inclusion/exclusion criteria for the group, 33 cases (11 from ADHD group, 17 from RD group, 4 from Control group) were excluded from analysis. Participants in the ADHD group were excluded primarily due to invalid eye movement data whereas participants in the RD group were primarily excluded due to failure to meet RD diagnostic criteria. A total of 78 subjects were included in the analysis.

In addition to a clinical diagnosis of ADHD, inclusion criteria for the ADHD group included performance in the average range on a brief measure of cognitive ability [24,25] and two reading tasks assessing phoneme awareness and reading comprehension [26]. Inclusion in the RD group required parent or teacher ratings in the average range on a measure of ADHD symptoms [27]. Given that many children with a read- ing disability display differences in performance between verbal and nonverbal cognitive ability tasks, inclusion criteria for the RD group required that children score in the average range on either the Verbal or Nonverbal composite score (i.e., rather than Composite IQ). Finally, inclusion criteria for children in the Control group included average or higher performance on a brief measure of cognitive ability (Composite IQ score) and both reading tasks as well as parent or teacher ratings below the 80th percentile on a measure of ADHD symptoms.

Overall, there were a similar number of male (55\%) and female (45\%) participants and no significant sex differences in the three groups. As expected, several children in the ADHD group (37\%) were taking medication for ADHD at the time they participated in the study. Lastly, the family income for children in the ADHD group was significantly lower than children in the RD and Control groups, likely due to differences in the referral source. The ADHD clinic accepts Medicaid payment whereas the READ clinic has a set outof-pocket fee of over $\$ 150$ and many Control group participants were children of faculty or staff at the university.

Table 1 presents group means for measures related to inclusion/exclusion criteria.

Composite IQ was lower for the RD group as compared to Control group participants; however the mean for all three groups was in the average range. As expected, the mean score for the ADHD and Control groups on the reading achievement subtests was in the average range, whereas the mean for the RD group was in the low average range. Although the majority of participants in the RD group had below average Performance average performance on the two reading subtests, there were several participants that met criteria for $\mathrm{RD}$ based on other measures in the RD test battery (i.e., performance was in the average range on the two Woodcock subtests). Likewise, the mean for the $\mathrm{RD}$ and Control groups was in the average (i.e., below clinical significance) range on the parent and teacher ADHD scale ratings.

\section{Procedures}

The study design and procedures were approved by the university's institutional review board. The assessment data collected through the ADHD Evaluation clinic and READ clinic were protected as required by HIPPA federal regulations. All participants were first administered the eye movement tasks, then a reaction time task, and a brief measure of cognitive ability and reading tasks if not 
Table 1. Group means for age and inclusion/exclusion measures.

\begin{tabular}{cccccccc}
\hline Measure & $\begin{array}{c}\text { ADHD } \\
(\mathrm{n}=27)\end{array}$ & $\begin{array}{c}\underline{\mathrm{RD}} \\
(\mathrm{n}=22) \\
\text { Mean SD }\end{array}$ & $\begin{array}{c}\text { Mean } S D \\
(\mathbf{n}=29)\end{array}$ & $F(2,75)$ \\
& Mean SD & \\
\hline WordAttack $^{\mathrm{a}}$ & 104.11 & 13.78 & 83.05 & 9.72 & 118.34 & 14.45 & 45.90 \\
PassComp $^{\mathrm{a}}$ & 102.41 & 13.36 & 83.64 & 11.59 & 117.86 & 12.61 & $43.52^{*}$ \\
CompIQ $^{\mathrm{a}}$ & $102.1 !$ & 12.94 & 93.41 & 13.91 & 111.62 & 10.87 & $13.42^{*}$ \\
ADHDParI $^{\mathrm{b}}$ & 94.87 & 6.66 & 65.45 & 30.30 & 36.31 & 26.04 & $45.62^{*}$ \\
ADHDParH $^{\mathrm{b}}$ & 86.11 & 23.14 & 52.86 & 31.35 & 40.00 & 28.74 & $20.23^{*}$ \\
ADHDParT $^{\mathrm{b}}$ & 94.68 & 4.90 & 63.07 & 29.69 & 40.33 & 28.29 & $44.07^{*}$ \\
ADHDTchI $^{\mathrm{b}}$ & 86.78 & 13.14 & 60.98 & 22.09 & 34.61 & 20.41 & $51.10^{*}$ \\
ADHDTchH $^{\mathrm{b}}$ & 83.00 & 12.63 & 51.70 & 27.44 & 27.21 & 20.99 & $47.97^{*}$ \\
ADHDTchT $^{\mathrm{b}}$ & 86.94 & 9.64 & 54.87 & 26.55 & 39.47 & 26.35 & $56.58^{*}$ \\
\hline
\end{tabular}

Note . PassComp = Passage Comprehension, CompIQ = Composite IQ, ADHDParI: Parent ADHD Inattentive Scale, ADHDParH $=$ Parent ADHD Hyperactive/Impulsive Scale, ADHDParT = Parent ADHD Total Scale, ADHDTchI $=$ Teacher ADHD Inattentive Scale, ADHDTchH $=$ Teacher ADHD Hyperactive/Impulsive Scale, ADHDTchT = Teacher

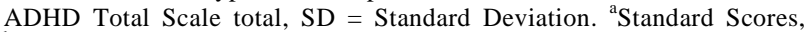
${ }^{\mathrm{b}}$ Percentiles; ${ }^{*} p<0.0001$

already administered. Children recruited from the READ Clinic were administered a battery of reading tasks as part of their initial assessment for the READ program.

Likewise, for ADHD children, a measure of cognitive ability and parent and teacher ADHD rating scales were completed during the initial evaluation for ADHD. Graduate and undergraduate research assistants were trained to administer all of the other research measures during two $1 \frac{1}{2}$ hour training sessions. All assessment materials included only the subject's individual identification number and necessary identifying information (e.g. date of birth, gender, and age).

\subsection{Eye Movement Measure}

The View Point Eye Tracker apparatus from Arrington Research was utilized to measure participants' eye movement patterns during a brief reading task. Participants were seated in front of a computer monitor and placed their heads in a chin and forehead rest, then instructed to watch the computer screen and move only their eyes and to keep their heads as still as possible. The reading task developed for this study required participants to read three sets of words, and five short sentences (1st grade reading level) that appeared on the computer screen. However, only eye movement data gathered from the five sentences was analyzed in this study. Each set of stimuli remained on the computer screen for approxi- mately three seconds. The eye movement task required 5-10 minutes to administer including adjustments and calibration; however, the actual reading task lasted less than one minute. At the end of the eye movement administration, subjects were asked questions to ensure that they actually read the sentences that were presented. The reliability of the eye movement tasks developed for this particular study is not known. The saccade threshold on the View Point Eye Tracker was set to 0.090 . This value represents the minimum velocity of an eye movement to be classified as a saccade. Moreover, saccades were considered vertical when the end point of the saccade was more than 45 degrees from where the saccade began. Eye movements that were slower than 0.090 were classified as fixations.

There were seven variables examined from the eye movement tracking task including average number of fixations, average fixation duration, average saccade duration, proportion of normal (i.e. left to right) saccades, proportion of regressive (i.e. right to left) saccades, proportion of vertical saccades, and total reading time. Average number of fixations was calculated for each child by summing the number of fixations across sentences and dividing by the total number of sentences. Likewise average fixation duration and average saccade duration were calculated by summing the total amount of time in milliseconds that the eye was engaged in a fixation/ saccade and dividing by the number of fixations/saccades for each sentence. The average durations were then summed and divided by the number of sentences. Proportion of left to right saccades, regressive saccades and vertical saccades were calculated by summing the number of each type of saccade across sentences, dividing by the total number of saccades and averaging across the sentences.

\section{Results}

\subsection{Preliminary Analyses}

Correlational analyses revealed several significant correlations between the eye movement variables. Specifically, read time and average number of fixations were correlated $(r=0.52)$ and proportion of sentence fixated upon and regressive saccades were inversely correlated $(\mathrm{r}=$ -0.48 ). Saccade duration and fixation duration were also inversely correlated $(r=-0.38)$. There was a strong negative correlation between the proportion of vertical saccades and the proportion of left to right saccades ( $\mathrm{r}=$ -0.78). There were no significant correlations between child age and the eye movement variables. Results of a MANOVA comparing eye movement performance for children taking/not taking medication in the ADHD 
group was non-significant ( $\mathrm{p}=0.61)$.

An ANOVA was also conducted comparing the three groups on the proportion of the sentence that was fixated. This information is important to consider because the frequency of the eye movement variables that will be examined in the main analyses are dependent upon the amount of the sentence that each child actually looked at. Results of the ANOVA were significant, F $(2,75)=6.67$, $\mathrm{p}=<0.01$. Post hoc analyses revealed that children in both the ADHD $(\mathrm{M}=0.75, \mathrm{SD}=0.24)$ and $\mathrm{RD}(\mathrm{M}=$ $0.73, \mathrm{SD}=0.24$ ) groups fixated on significantly less of the sentences than children in the Control group $(\mathrm{M}=$ 0.93, SD = 0.14). However, there were no significant differences between groups in regards to correct answers to questions about the eye movement sentences, $\mathrm{F}(2,61)$ $=0.61, \mathrm{p}=0.55$. In addition, previous research has found that there is a great deal of variability in the percentage of words that a reader fixates and that a typical reader will skip between $20-40 \%$ of the words in a sentence (e.g., Paulson, 2002).

\subsection{Group Differences in Eye Movement}

Results of a MANOVA, grouping by ADHD, RD, and Control, and entering the seven eye movement variables was significant $\mathrm{F}(7,70)=4.74, \mathrm{p}<0.001$. As seen in Table 2, univariate analyses revealed significant group differences for all eye movement variables with the exception of saccade duration. Children in both the ADHD and RD groups displayed a lower proportion of left to right saccades and higher proportion of regressive saccades as compared to the Control group. There was a significant difference between the RD and Control group for average number of fixations (greater for RD group). Children in the ADHD group also displayed a greater proportion of vertical saccades as compared to the Control group. Finally, there was a significant difference between the RD group and the ADHD and Control groups for total read time and fixation duration ( $\mathrm{RD}>$ ADHD and Control for both).

In order to determine which eye movement variables best distinguish between children in the ADHD and RD groups, a stepwise discriminant analysis was conducted, entering average number of fixations, reading time, fixation duration, proportion of left to right saccades, proportion of regressive saccades, and proportion of vertical saccades as predictors. In the first step of the analysis, proportion of left to right saccades entered as a significant predictor (Wilkes $=0.78, \mathrm{p}<0.0001$ ), followed by total reading time (Wilkes $=0.62, \mathrm{p}<0.001$ ). Based on these two predictors, $57.7 \%$ of cases were correctly classified with the Control group having the highest rate of correct classification (79\%), followed by the RD group
(59\%). The lowest rate of correct classification was for the ADHD group (33\%).

\section{Discussion}

The current study was undertaken in order to examine whether eye movement tracking could be used as a reliable means of differentiating between children with ADHD and RD. Overall, results yielded minimal support for differences between ADHD and RD children in terms of eye movement. Children in both the ADHD and RD groups displayed significantly shorter fixations and a lower proportion of left to right saccades as compared to the Control group. The one significant difference between the ADHD and RD group was in total reading time. As predicted, children in the RD group displayed slower reading than children in the ADHD and Control group. Thus, there is some potential for the combination of left to right saccades and total reading time to differentiate between children with ADHD and RD. However, further research is needed to determine support for clinical utility of eye movement as an assessment measure given that no more than half of the RD and ADHD children were correctly classified based on select eye movement variables.

Consistent with previous research, children with RD displayed longer fixations and slower reading speed as compared to Controls [18]. Examining the eye movement patterns of children with ADHD on reading tasks is a relatively new area of study. Therefore, it is difficult to determine whether the results of the current study suggest-

Table 2. Eye movement variables for Attention Deficit/Hyperactivity Disorder (ADHD), Reading Disorder (RD), and Control groups.

\begin{tabular}{cccccccc}
\hline & \multicolumn{2}{c}{ ADHD } & \multicolumn{2}{c}{$\underline{\mathrm{RD}}$} & \multicolumn{2}{c}{$\underline{\text { Control }}$} & \\
DV & \multicolumn{2}{c}{$(\mathrm{n}=27)$} & \multicolumn{2}{c}{$(\mathrm{n}=22)$} & \multicolumn{2}{c}{$(\mathrm{n}=29)$} & $F(2,73)$ \\
& Mean & SD & Mean & SD & Mean & SD & \\
\hline \# Fixations & 7.45 & 1.80 & $8.34^{\mathrm{a}}$ & 2.15 & $6.94^{\mathrm{b}}$ & 1.43 & $3.81^{*}$ \\
Fix Dur(ms) & $245.88^{\mathrm{b}}$ & 98.15 & $350.55^{\mathrm{a}}$ & 181.54 & $238.89^{\mathrm{b}}$ & 78.62 & $5.13^{*}$ \\
Sac Dur(ms) & 81.99 & 28.70 & 75.61 & 13.43 & 81.80 & 34.51 & 0.39 \\
\% LR & $0.68^{\mathrm{a}}$ & 0.26 & $0.71^{\mathrm{a}}$ & 0.23 & $0.91^{\mathrm{b}}$ & 0.08 & $10.84^{* *}$ \\
\% Reg & $0.13^{\mathrm{a}}$ & 0.10 & $0.15^{\mathrm{a}}$ & 0.10 & $0.07^{\mathrm{b}}$ & 0.06 & $6.58^{*}$ \\
\%Vertical & $0.12^{\mathrm{a}}$ & 0.17 & 0.10 & 0.18 & $0.01^{\mathrm{b}}$ & 0.03 & $4.75^{*}$ \\
Time(s) & $2.30^{\mathrm{a}}$ & 0.666 & $3.02^{\mathrm{b}}$ & $0.737^{\mathrm{b}}$ & $2.16^{\mathrm{a}}$ & 0.701 & $10.58^{* *}$ \\
\hline
\end{tabular}

Note. \# Fixations = Average number of fixations, Fix Dur(ms) = Fixation duration in milliseconds, Sac Dur = Saccade duration in milliseconds, \% LR = Proportion of left to right saccades, \% Reg = Proportion of regressions, Time $=$ Total read time. SD $=$ Standard Deviation. DV = Dependent Variable. Different superscripts indicate significant group difference $(p<0.05)$; ${ }^{*} p<0.01 ; * * p<0.001$ 
ing a significantly lower proportion of left to right saccades in ADHD children compared to children in a Control group is characteristic of all children with ADHD or whether this finding is specific to the current study. Overall, results of this study suggest that eye movement performance of children with ADHD is more similar than dissimilar to children with RD with the exception of total reading time. One possible explanation for the lack of difference between the ADHD and RD groups might involve the number of children eliminated from the study due to excessive head and body movements which perhaps resulted in less variability.

Another limitation that may have contributed to the lack of difference in eye movement between the two clinical groups is subthreshold comorbidity. Children in the ADHD group may have had a developing reading disability that was not evident in the two Woodcock reading tasks. Likewise, some of the children in the RD group may have had subthreshold traits of ADHD. A significant level of subthreshold comorbidity could have contributed to greater variability within groups and therefore decreased likelihood of significant differences between groups. Given the significant rate of comorbidity between ADHD and RD, future research may compare the eye movement patterns of children in a comorbid ADHD+RD group to children with either ADHD or $\mathrm{RD}$ alone.

Finally, the eye movement task used in this study might be a limitation. Calibration sensitivity and lack of a "bite bar" to keep children's heads still contributed to lost data and perhaps less valid data in some cases, as some vertical eye movements may have been due to sensitivity to movement. A second possible limitation is the reading task itself, which was very brief and may not have been challenging enough for older children. Garzia et al. [15] suggested that the types of eye movements made by readers are significantly influenced by the qualities of the individual words and overall text. Greater variability in eye movement may have been observed during a longer and/or more challenging reading task. Children in the ADHD group also fixated on a significantly lower proportion of the sentences than children in the Control group, which may have had some effect on the patterns of eye movements observed. However, this did not appear to affect the comprehension of children in the ADHD group, as there were no significant differences between any of the groups on sentence comprehension.

Further investigation is needed to determine if eye movement tracking may be useful in distinguishing between ADHD and RD children that display reading difficulties. Given the results of the present study, future research may specifically consider proportion of left to right saccades and average reading time as these variables demonstrated that greatest potential for differentiating between groups.

Given that the present results provided minimal support for eye movement variables to distinguish between clinical groups (i.e. ADHD and RD groups), it is premature at this time to suggest use of eye movement measures in assessment batteries. Also, given the significant percentage of children in the present study that produced invalid results due to excessive movement, use of eye movement tasks with ADHD children, particularly those with significant hyperactivity, may be of minimal benefit. A few of the children with ADHD in the present study also expressed a strong dislike for the eye movement task. Therefore, unless further research finds greater support for eye movement to discriminate between clinical groups, the costs associated with this measure may exceed the benefits.

\section{Acknowledgements}

This research was partially supported by a grant from the Office of Information Technology at Indiana State University.

\section{REFERENCES}

[1] American Psychiatric Association, "Diagnostic and Statistical Manual of Mental Disorders,” 4th Edition, Text Revision, Washington, DC, 2000.

[2] J. R. Nelson, G. J. Benner, K. Lane and B. W. Smith, "Academic Achievement of K-12 Students with Emotional and Behavioral Disorders,” Exceptional Children, Vol. 71, No. 1, 2004, pp. 59-73.

[3] K. Rayner, "Eye Movements in Reading and Information Processing: 20 Years of Research,” Psychological Bulletin, Vol. 124, No. 3, 1998, pp. 372-422.

[4] R. A. Barkley, "Response Inhibition in Attention-Deficit Hyperactivity Disorder,” Mental Retardation and Developmental Disabilities, Vol. 5, No. 3, 1999, pp. 177-184.

[5] A. M. LaGreca, A. F. Kuttler and W. L. Stone, "Assessing Children through Interviews and Behavioral Observations,” In: C. E. Walker and M. C. Roberts, Eds., Handbook of Clinical Child Psychology, 3rd Edition, New York, John Wiley \& Sons, 2001, pp. 90-106.

[6] S. M. Bell, R. S. McCallum and E. A. Cox, "Toward a Research Based Assessment of Dyslexia: Using Cognitive Measures to Identify Reading Disabilities,” Journal of Learning Disabilities, Vol. 36, No. 6, 2003, pp. 505516.

[7] V. A. Berninger, “Understanding the 'Lexia' in Dyslexia: A Multidisciplinary Team Approach to Learning Disabilities," Annals of Dyslexia, Vol. 51, No. 1, 2001, pp. 23-48. 
[8] E. G. Willcutt, B. F. Pennington, R. Boada, J. S. Ogline, R. A. Tunick, N. A. Chhabildas and R. K. Olson, "A Comparison of the Cognitive Deficits in Reading Disability and Attention-Deficit/Hyperactivity Disorder," Journal of Abnormal Psychology, Vol. 110, No. 1, 2001, pp. 157-172.

[9] R. I. Nicolson and A. J. Fawcett, "Development of Objective Procedures for Screening and Assessment of Dyslexic Students in Higher Education,” Journal of Research in Reading, Vol. 20, No. 1, 1997, pp. 77-83.

[10] R. A. Barkley, "Behavioral Inhibition, Sustained Attention, and Executive Functions: Constructing a Unifying Theory of ADHD,” Psychological Bulletin, Vol. 121, No. 1, 1997, pp. 65-94.

[11] S. Y. Padget, D. F. Knight and D. J. Sawyer, "Tennessee meets the Challenge of Dyslexia," Annals of Dyslexia, Vol. 46, No. 1, 1996, pp. 51-72.

[12] S. L. Decker, D. E. McIntosh, A. M. Kelly, S. K. Nicholls and R. S. Dean, "Comorbidity among Individuals Classified with Attention Disorders," International Journal of Neuroscience, Vol. 110, No. 1-2, 2001, pp. 43-54.

[13] A. T. Duchowski, "A Breadth-First Survey of Eye Tracking Applications," Behavior Research Methods, Instruments, \& Computers, Vol. 34, No. 4, 2002, pp. 455-470.

[14] G. F. Eden, J. F. Stein, M. H. Wood and F. B. Wood, "Differences in Eye Movements and Reading Problems in Dyslexic and Normal Children,” Vision Research, Vol. 34, No. 10, 1994, pp. 1345-1358.

[15] R. P. Garzia, J. E. Richman, S. B. Nicholson and C. S. Gaines, "A New Visual-Verbal Saccade Test: The Developmental Eye Movement Test (DEM)," Journal of the American Optometric Association, Vol. 61, No. 2, 1989, pp. 124- 135.

[16] R. Kliegl and R. Engbert, "Fixation Durations before Word Skipping in Reading," Psychonomic Bulletin \& Review, Vol. 12, No. 1, 2005, pp. 132-138.

[17] D. Adler-Grinberg and L. Stark, "Eye Movement, Scan Paths, and Dyslexia," American Journal of Optometry and Physiological Optics, Vol. 55, No. 8, 1978, pp. 557-570.
[18] S. Hawelka and H. Wimmer, "Impaired Visual Processing of Multi-Element Arrays is Associated with Increased Number of Eye Movements in Dyslexic Reading," Vision Research, Vol. 45, No. 1, 2005, pp. 855-863.

[19] D. P. Munoz, I. T. Armstrong, K. A. Hampton and K. D. Moore, "Altered Control of Visual Fixation and Saccadic Eye Movements in Attention-Deficit Hyperactivity Disorder,” Journal of Neurophysiology, Vol. 90, No. 1, 2003, pp. 503-515.

[20] D. Feifel, R. H. Farber, B. A. Clementz, W. Perry and L. Anllo-Vento, "Inhibitory Deficits in Ocular Motor Behavior in Adults with Attention-Deficit Hyperactivity Disorder,” Biological Psychiatry, Vol. 56, No. 5, 2004, pp. 333-340.

[21] T. D. Gould, T. M. Bastain, M. E. Israel, D. W. Hommer and F. X. Castellanos, "Altered Performance on an Ocular Fixation Task in Attention-Deficit/Hyperactivity Disorder," Biological Psychiatry, Vol. 50, No. 8, 2001, pp. 633-635.

[22] C. H. Klein, A. Raschke and A. Brandenbusch, "Development of Pro- and Antisaccades in Children with Attention-Deficit Hyperactivity Disorder (ADHD) and Healthy Controls,” Psychophysiology, Vol. 40, No. 1, 2003, pp. 17-28.

[23] S. H. Mostofsky, A. G. Lasker and L. E. Cutting, "Oculomotor Abnormalities in Attention Deficit Hyperactivity Disorder: A Preliminary Study,” Neurology, Vol. 57, No. 3, 2001, pp. 423-430.

[24] D. Wechsler, "Wechsler Abbreviated Scale of Intelligence," The Psychological Corporation, San Antonio, 1999.

[25] A. S. Kaufman and N. L. Kaufman, "Kaufman Brief Intelligence Test,” 2nd Edition, Circle Pines, AGS Publishing, 2004.

[26] R. W. Woodcock, "Woodcock Language Proficiency Battery Revised,” Riverside Publishing, Itasca, 1991.

[27] G. J. DuPaul, T. J. Power, A. D. Anastopoulos and R. Reid, "ADHD Rating Scale-IV: Checklists, Norms, and Clinical Interpretation,” Guilford Press, New York, 1998. 